\title{
Disinhibition of return: Unnecessary and unlikely
}

\author{
RAYMOND M. KLEIN and WILLIAM C. SCHMIDT \\ Dalhousie University, Halifax, Nova Scotia, Canada \\ and \\ HERMANN J. MÜLLER \\ Birbeck College, University of London, London, England
}

\begin{abstract}
Recently, from data obtained with a temporal order judgment (TOJ) task, Gibson and Egeth (1994) concluded that inhibition of return (IOR; a response time effect that reveals slower responding to targets at previously cued versus uncued locations) reflects impaired perceptual processing. By replotting their data, we demonstrate that the perception of temporal order is influenced only by the facilitatory effect of a cue at short stimulus onset asynchronies (SOAs) and is unaffected by IOR at long SOAs. The target paper proposed that, when extra stimuli are presented at task-relevant locations (i.e., in the TOJ task), IOR is prevented by a hypothetical process that is known as disinhibition of return (DOR). We argue that the assumptions that IOR affects perceptual processing and that DOR exists are unnecessary, as a more parsimonious response-based interpretation of IOR is consistent with their data. Further, we summarize recent results and present new data that demonstrate that DOR is unlikely.
\end{abstract}

Inhibition of Return (IOR) is operationally defined as a slower response time (RT; manually or with eye movements) to a target presented at a previously cued location than at a previously uncued location (Posner \& Cohen, 1984; Taylor \& Klein, in press). This inhibitory effect is commonly observed to set in approximately $300 \mathrm{msec}$ after cue onset, before which people generally respond faster to a target at the cued location (i.e., there is an expected attentional cuing effect). To explain this pattern of results, Posner and Cohen postulated that visual attention was inhibited from returning to a location from which it had been recently removed. Recent research has focused on whether the source of IOR is inhibited perceptual processing, inhibited motoric response, or a combination of perceptual and motor factors. In an effort to determine whether IOR involves inhibited perceptual representations, a number of investigators have sought evidence on the basis of purely perceptual measures (Gibson \& Egeth, 1994; Kwak, 1992; Maylor, 1985; Posner, Rafal, Choate, \& Vaughan, 1985; Schmidt, 1996). The rationale is straightforward: If IOR results primarily from response or motor processing, perceptual judgments with no motor component should show little or no effect of IOR.

Maylor (1985) was the first to use a perceptual measure of processing to investigate IOR. Maylor reasoned that,

This research was supported by a NSERC Canada operating grant to R.K., by NSERC Canada Post Graduate funding and an Honrary Izaak Walton Killam Scholarship to W.C.S., and by SERC Grant GR/H/ 54966 to H.J.M. Thanks to the reviewers, Tram Neill, Howard Egeth, and particularly Bradley Gibson, for their comments on previous versions of this manuscript. Correspondence concerning this paper should be addressed to R. M. Klein, Department of Psychology, Dalhousie University, Halifax, NS, Canada B3H 4J! (e-mail: klein@or.psychology. dal.ca). if IOR involves the inhibition of perceptual signal transmission from a previously attended (or stimulated) location, it ought to be possible to observe the modulatory effects of IOR with a purely perceptually based dependent measure, rather than with simple RT, which is likely to reflect response-based processing in addition to components arising from a perceptual locus. Maylor chose to examine IOR through the use of temporal order judgments (TOJs).

TOJs are reports that observers make about which of two stimuli at disparate spatial locations is perceived to be presented first in time. Current thinking holds that the orienting of visual attention has the modulatory effect of speeding the transmission of attended signals in the visual system. The effects of covert orienting on the perceived order of signal arrival has been demonstrated on a number of occasions (see Sternberg \& Knoll, 1973; also see subsequent work by Hikosaka, Miyauchi, \& Shimojo, 1993; Stelmach \& Herdman, 1991).

Maylor's (1985) TOJ data and the data of other studies in which TOJs in the IOR paradigm are examined (Gibson \& Egeth, 1994; Kwak, 1992; Posner et al., 1985) have demonstrated that, at long cue-target intervals during IOR exerts its effects on the RT, there is no indication of delayed signal transmission at previously cued locations. Rather, with TOJs as a dependent measure, there is evidence for speeded signal transmission at short cue-target intervals, as would be expected from the facilitative effects of orienting, and this facilitation diminishes toward zero as the cue-target interval is lengthened. That her TOJ data did not coincide with IOR effects observed with RT measures led Maylor to conclude that IOR reflects response, or motoric, inhibition, rather than a delay arising from perceptual impairment. Posner et al. reached a similar conclusion when they compared performance on a TOJ task with eye-movement responses to the same TOJ 
stimuli. Whereas the subjects in the eye movement task, who were instructed to saccade to stimuli as they pleased, showed a bias against orienting toward the cue, there was no evidence-for delayed perception of these cued stimuli in the TOJ task.

\section{The Claims of Gibson and Egeth}

Recently in this journal, Gibson and Egeth (1994) presented a series of experiments that used a TOJ task to reexamine whether IOR affects perceptual processing. Their methods differed from Maylor's (1985) only in that RT data were collected along with TOJs. Because of the importance assigned to speed, the task will be referred to as a speeded TOJ task.

Gibson and Egeth (1994) attempted to reconcile the notion that IOR affects perceptual processing with their own and others' TOJ results that failed to support this idea. They did so through a creative and complex line of argument, based primarily on their RT data, that included the postulate of a novel process they called disinhibition of return (DOR). As stated in their abstract, they concluded that IOR affects perceptual processing and that IOR affects TOJs in some conditions but not in others. These statements were reinforced in the paper's concluding section, where it is stated that "the present findings are important because they are the first to show that IOR does affect TOJs, at least in certain situations" (p. 677) and that "this study was conducted to clarify the functional nature of IOR. In particular, we investigated whether IOR could be shown to affect TOJs under the assumption that such an effect would reflect an impairment in perceptual processing at the cued location (Maylor, 1985). Affirmative evidence was obtained, thus strengthening Abrams and Dobkin's (1994) contention that IOR reflects, at least in part, a perceptual impairment" (p. 679).

Stated simply and without the qualifications inherent in the balance of the paper, these conclusions are too easily misinterpreted. Even qualified, however, these are not so much conclusions implied by the data as they are a reflection of Gibson and Egeth's (1994) foundational assumptions, combined with a novel interpretation of the findings and a reinterpretation of other TOJ work.

In this commentary, we first illustrate that the psychophysical (judgment) data collected in Gibson and Egeth's (1994) speeded TOJ task provide a rigorous replication of Maylor's (1985) work, despite the introduction of an instructional difference. Then, after outlining the intricate reasoning from their RT data, which led Gibson and Egeth to propose the existence of DOR, we show that response-based interpretations of IOR are not refuted by Gibson and Egeth's findings. Finally, we present situations that fail to empirically support the operation of a process such as DOR. We conclude that DOR was unnecessary for the explanation of the RT data in the target paper, that DOR is unlikely, and that the assertion that IOR is manifest in delayed perceptual processing is unwarranted on the basis of the evidence presented by Gibson and Egeth.

\section{Does IOR Affect TOJs?}

As was noted earlier, TOJ methodology involves asking subjects to decide which of two stimuli appeared first. Instead of allowing subjects time to reflect on perceptual experiences before indicating their judgment (as in previous studies), Gibson and Egeth (1994), seeking to more closely approximate the conditions in which IOR has typically been observed, requested that their subjects make speeded responses. Because IOR is apparent when speeded responses are made to a single stimulus in a detection or localization task, it was possible that IOR might be observed in such a speeded TOJ task.

Figure 1 presents the trial sequence used in Gibson and Egeth's (1994) TOJ task. A $100 \mathrm{msec}$ cue (the $100 \mathrm{msec}$ brightening of one of two squares that were situated either to the left or to the right of a fixation cross) was followed, after a cue lead time (stimulus onset asynchrony [SOA] between the cue and the target) that ranged from 100 to

\section{First-cued condition}

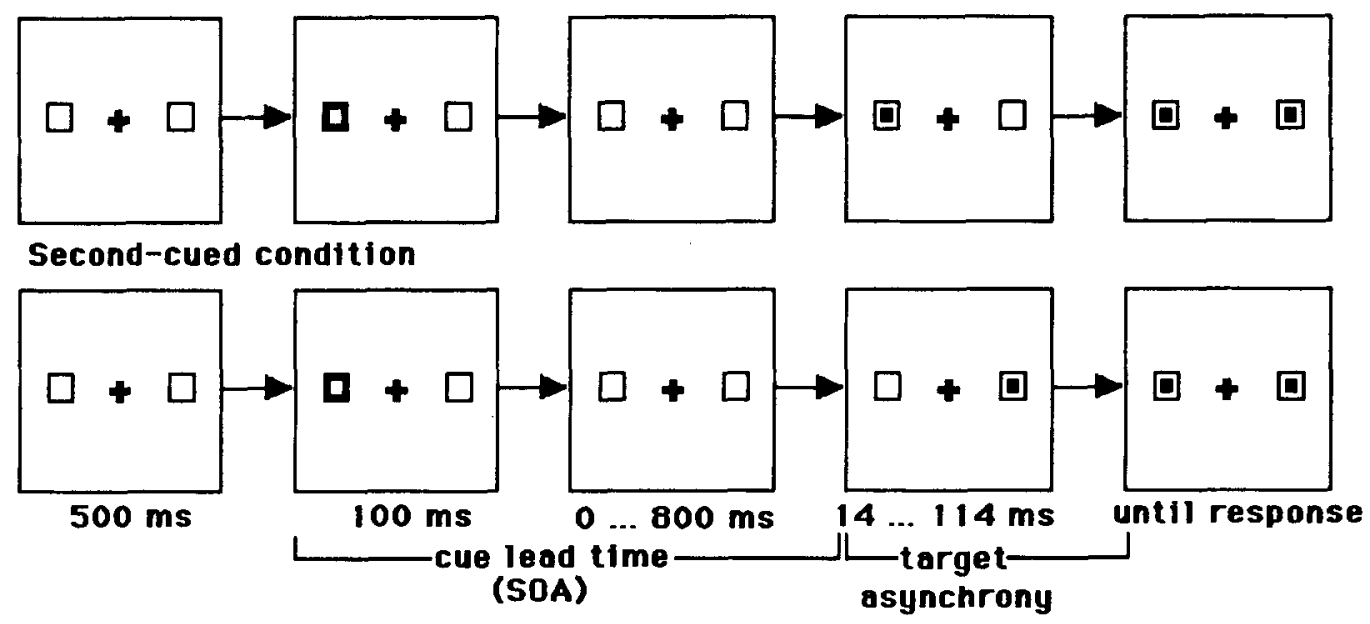

Figure 1. Experimental trial sequence used by Gibson and Egeth (1994). See the text for details. 
$900 \mathrm{msec}$, by a luminance onset target (the filled square appearing inside the larger square) at either the location of the cue (the first-cued condition) or at an uncued location (the second-cued condition). These two conditions were equiprobable; hence, such a cue is uninformative concerning the location of the first target. After a second temporal interval (the target asynchrony, which was varied between 14 and $114 \mathrm{msec}$ ), a second stimulus, identical to the target, appeared in the unoccupied square opposite to that of the first target. The subject's task was to press a button that corresponded to the location of the first perceived target stimulus as quickly as possible.

In presenting the results of Experiment 1 , Gibson and Egeth (1994) note that they replicated the absence of an effect of IOR upon TOJs: "Importantly, however, there was no evidence that TOJs were influenced by IOR following long cue lead times" (p. 672). However, because of their claim that IOR affects TOJs, coupled with their complex interpretations of follow-up experiments and a method of data presentation that obscures the psychophysical relationship between stimulus asynchrony and perception of temporal order, their acknowledged replication and extension of Maylor (1985) may not be obvious, even to knowledgeable readers. For these reasons, these valuable data on TOJs warrant greater emphasis and a fuller presentation than they have so far received. In this section, we describe, in some detail, the methods and rationale used and the findings obtained when cuing and TOJs are combined, in order to address the question, Does IOR affect the perceived order of stimuli presented closely spaced in time?

Suppose that targets are presented at separate locations, with variation in temporal asynchrony, and that an observer's task is to report which appeared first. If both items are presented simultaneously (the target asynchrony is zero), then, in general, observers report with approximately the same frequency that each item occurred first (this is known as the point of subjective simultaneity, or PSS). With an increasing target asynchrony, the first target to appear is more often reported as occurring first. Figure 2 presents hypothetical data for this baseline condition. When target $\mathrm{A}$ leads target $\mathrm{B}$, subjects frequently report that $A$ did occur first. When $A$ lags behind $B$, there are fewer reports that $A$ appeared first.

Next, consider the pattern of data from a manipulation that decreases signal transmission time from one location

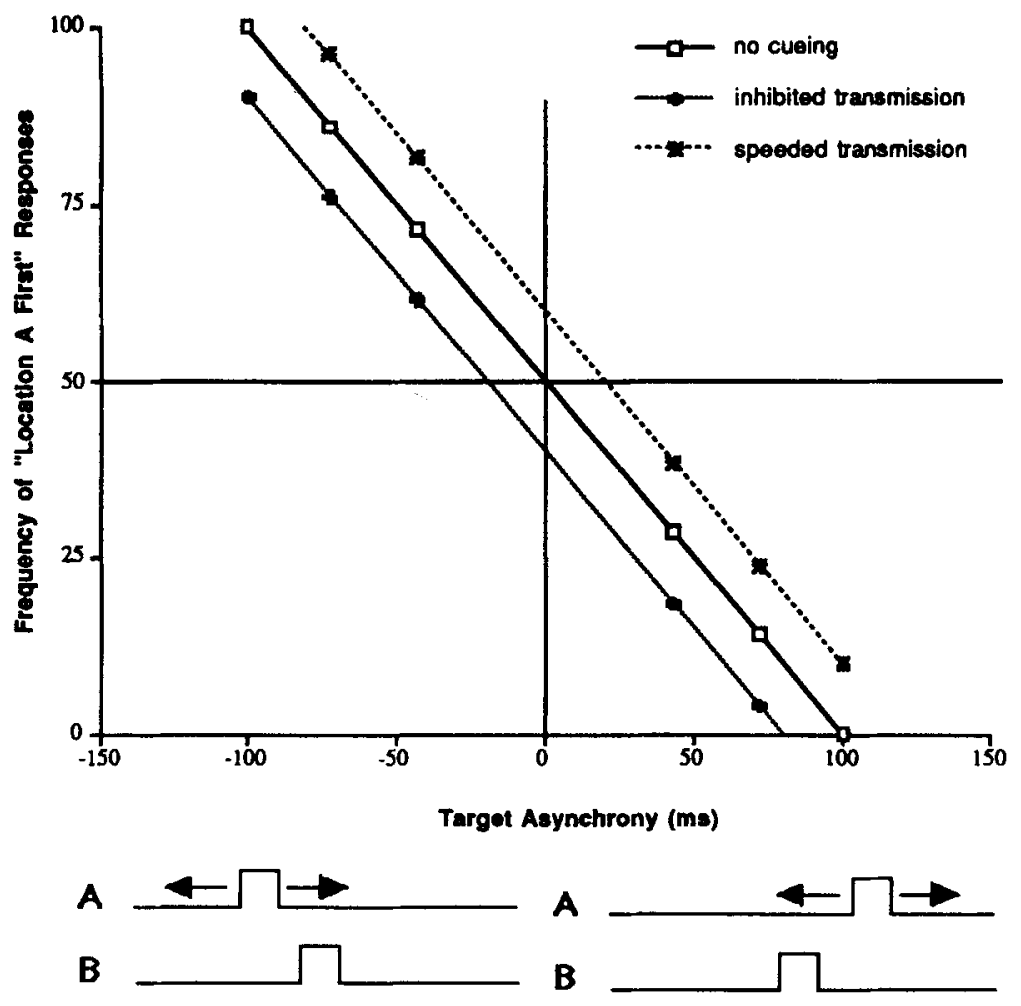

Figure 2. Ideal temporal order judgment data under conditions of no cuing (baseline), location $A$ inhibited, and location $A$ facilitated (arbitrarily, we have used negative target asynchronies to represent $A$ before $B$ ). The timelines beneath the graph illustrate, on the left, the case in which location $A$ is stimulated before location $B$ and, on the right, the case in which $B$ occurs before $A$. The arrows show possible facilitatory (left arrow) and inhibitory (right arrow) effects of cuing the location of $A$ on the perceived time of such stimulation. 


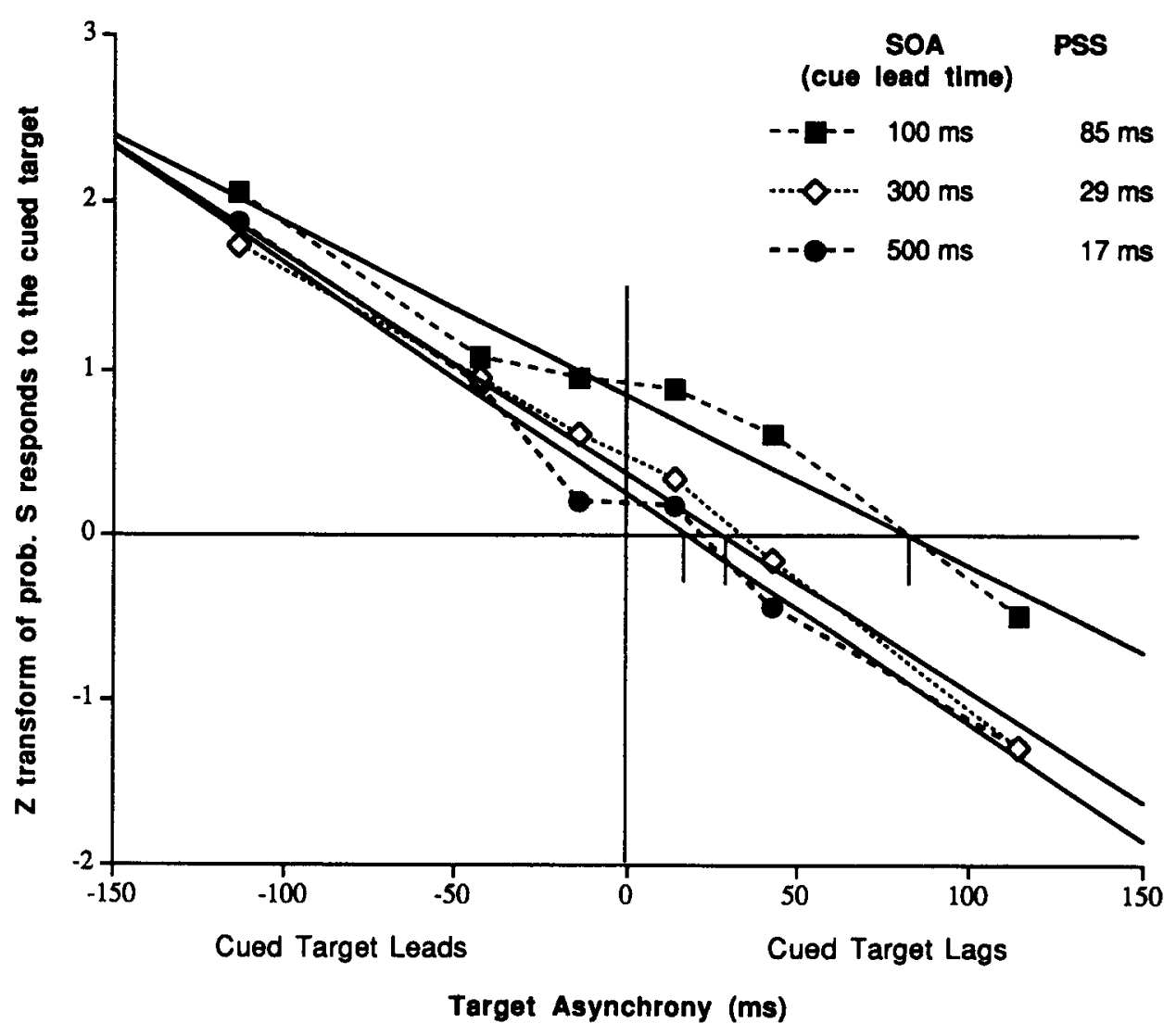

Figure 3. Gibson and Egeth's (1994) Experiment 1 data, transformed and plotted as a function of target asynchrony. The presented linear regression functions (solid lines) were derived from the data in order to recover point of subject simultaneity values, which are marked by vertical bars at each regression line's $x$ intercept.

(such as the speeding of signal transmission at location A by the orienting of attention toward that location by a recent cue). In the present context, such a facilitatory effect on processing speed will modify TOJs (see the Location A Facilitated data in Figure 2). Signals arising from near the cued location will arrive for subsequent processing earlier than they would have in the baseline condition. That is, the function in Figure 2, is shifted rightwards from the baseline function and, consequently, so is the subject's PSS. When the stimulus at the cued location really leads the other item, the decrease in signal transmission time will cause the former to be perceived as occurring first over a wider range of target asynchronies. Conversely, when the stimulus at the cued location lags the other stimulus, at very short lags it may, because of the facilitation, be perceived to occur first.

The pattern of effects reported by Maylor (1985), using uninformative peripheral cues (see Figure 1), and by Stelmach and Herdman (1991), using a cuing method whereby observers were directed to attend to peripheral spatial locations, matches the above description precisely at short cue target SOAs, which suggests that facilitative effects result from attentional cuing manipulations. If IOR acts to delay perceptual processing (increase or delay trans- mission time), then uninformative cues, combined with longer cue-target SOAs, should shift the PSS in the opposite direction from that in the facilitative case (see the Location A Inhibited data in Figure 2). Such a shift was not observed by Maylor or by Posner et al. (1985).

By replotting the Gibson and Egeth (1994) TOJ data at each level of cue lead time, with the target asynchrony along the abscissa and the probability that the subject responded to the cued target location on the ordinate, it can be seen that, regardless of the cue lead time, neither the function nor the PSS has been shifted in a manner consistent with IOR's causing a delay in signal transmission. ${ }^{1}$ Figures 3 and 4 present the $Z$ transform of the data from Gibson and Egeth's Experiments 1 and 3, respectively. The $Z$ scores, which were standardized normal scores, transform ogival functions (typical of such psychometric data) into linear functions, from which PSS values can be recovered by simple linear regression. Figures 3 and 4 also present the regression functions for each condition, with the PSS (the $x$ intercept) marked by vertical bars.

The PSS values recovered from Gibson and Egeth's (1994) experiments are presented in Figure 5, together with Maylor's (1985) original data. It is clear that, despite the emphasis on speed of responding in addition to 


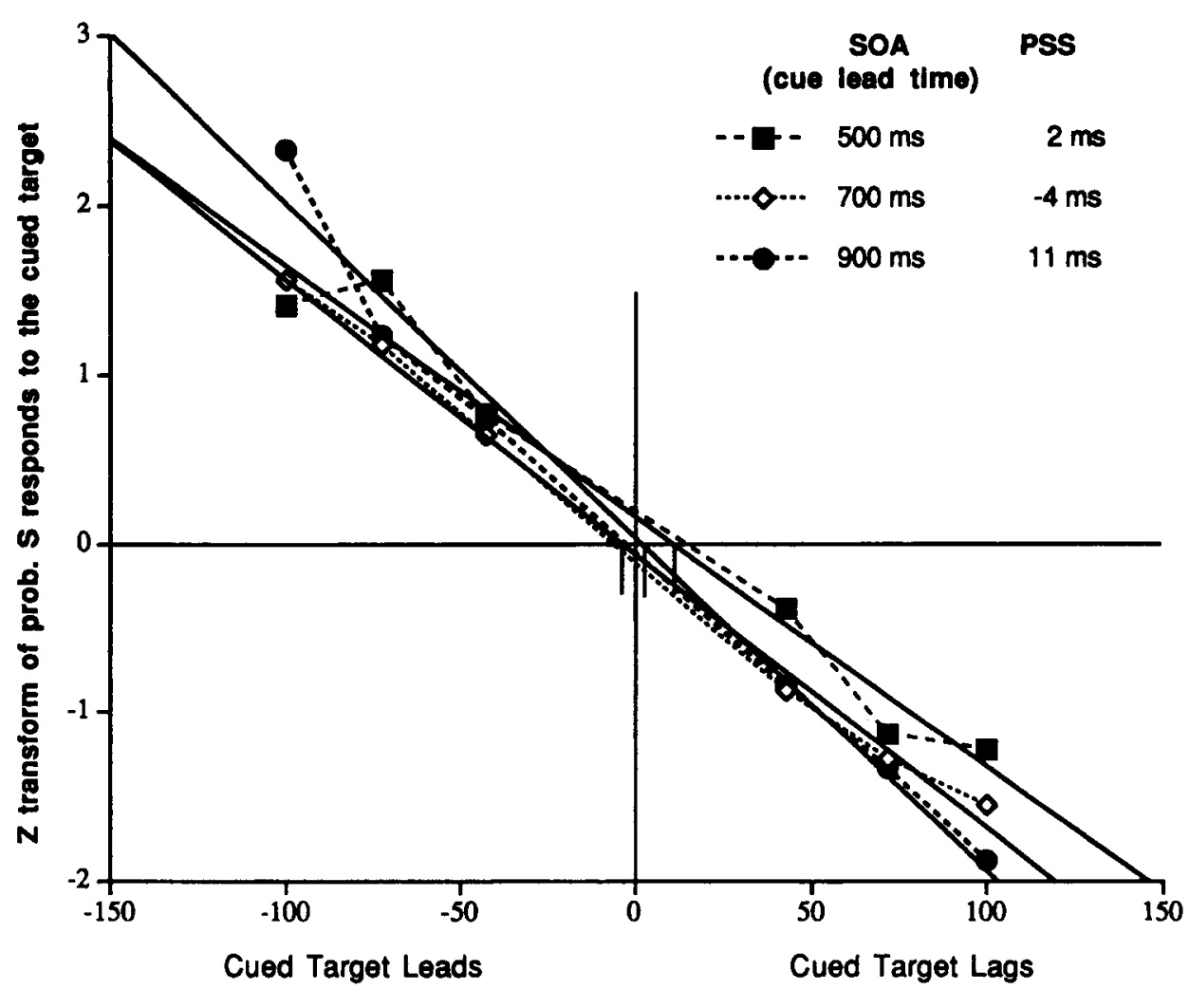

Target Asynchrony (ms)

Figure 4. Gibson and Egeth's (1994) Experiment 3 data, transformed and plotted as a function of target asynchrony, as in Figure 3. Solid lines represent linear regression functions computed to recover point of subjective simultaneity values described in the text.

accuracy, the Gibson and Egeth paradigm provides a clear replication of Maylor. Maylor's finding is often regarded skeptically, because she tested only 2 subjects, including herself. Considering the change in instructional set (speeded responses), the Gibson and Egeth replication with 22 naive subjects provides an especially impressive demonstration of the robustness of this result. In all cases, the PSS values are largest at the $100 \mathrm{msec}$ cue lead time and decrease monotonically toward zero as this interval increases. They are not consistently negative at any cue-target SOA, showing that IOR does not slow or delay the arrival time of targets, even when these are presented long enough after a cue that detection or localization RTs would have been slowed. Returning to the question of whether IOR affect judgments of temporal order, the answer is, clearly, that it does not. Gibson and Egeth acknowledge this (p. 672), but, as noted earlier, statements made in more salient locations of the paper could easily lead the reader to a different conclusion.

Although the natural interpretation of this finding is that IOR does not affect perception (in the sense of delaying arrival times), Gibson and Egeth's (1994) finding that IOR was sometimes observed in RT in this paradigm (when the two targets were temporally far enough apart to avoid temporal order errors) led them to hypothesize that IOR was released at short target asynchronies and to suggest that "IOR may never be shown to affect accuracy in the TOJ task" (p. 677), which depends on short intertarget delivery times. This challenge to the viability of the TOJ task as a tool for exploring whether IOR affects perception depends critically on Gibson and Egeth's proposal that IOR is canceled by DOR.

\section{The Role of Disinhibition of Return}

Experiment 1 of Gibson and Egeth (1994) included single-target trials, in addition to the double-target trials discussed in the preceding section. Single-target trials were identical to conventional IOR paradigms: a cue appeared, followed by a target either at the same location as the cue or in the opposite hemifield. In double-target trials, a second stimulus, presented at the unoccupied location, followed the target after a variable SOA, called the target asynchrony (see Figure 1). The observer's task was to make a speeded localization response that corresponded to the position of the first target.

As expected, RTs on single-target trials were affected by IOR, showing both an early-acting facilitation and a late-acting inhibition of responding to the location of the 


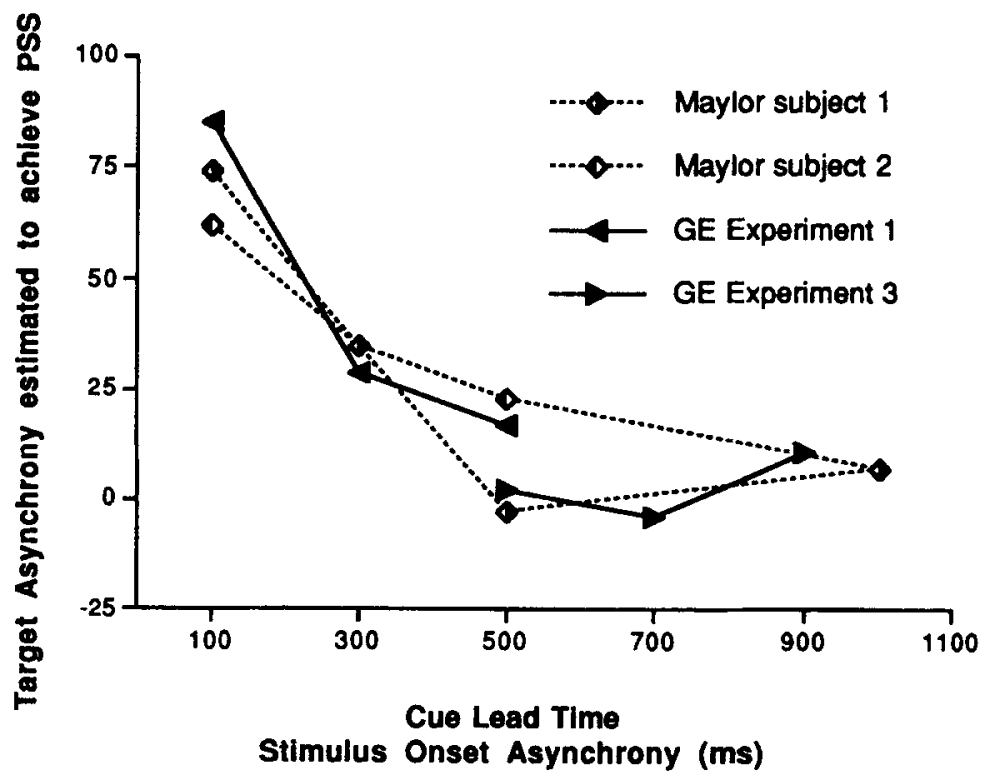

Figure 5. Point of subjective simultaneity as a function of stimulus onset asynchrony, from Maylor (1985; Experiment 4) and Gibson and Egeth (1994, Experiments 1 and 3).

cued single target. On double-target (TOJ) trials in which the first target appeared at the cued location (the firstcued condition), RT was faster than it was on trials in which the target appeared at the location opposite to the cued location (the second-cued condition). These RT data (see Figure 2 of Gibson \& Egeth, 1994) reveal the facilitative influence of the cue in speeding the transmission of signals in the visual system. The accompanying TOJ data corroborated this pattern of results: A greater frequency of errors in TOJs was made in the second-cued condition than in the first-cued condition. Apparently, the cue had the effect of speeding signals in the visual system, resulting in the perception that the target at the cued location appeared first, regardless of whether this was (firstcued trials) or was not (second-cued trials) the case.

It was concluded that IOR was operating in the first experiment because single-target trials, which essentially replicated the conventional IOR paradigm, demonstrated the expected pattern of RTs. However, in keeping with the general findings that IOR affects RT but not judgments of temporal order, the cue did not appear to delay the arrival times of targets at the cued location on doubletarget trials.

In considering the question of why the usual pattern of RT effects indicative of IOR (slower responding at previously cued than at uncued locations) was not detected in double-target trials (as well as the question of why IOR was not apparent in TOJs), Gibson and Egeth (1994) assumed that IOR was operating in the first-cued condition, and they hypothesized that IOR at the cued location in the second-cued condition may have been prevented, or altered, by the appearance of the first target stimulus. Lingering facilitation at the cued location in this condi- tion could accelerate the second target's arrival, thus decreasing the difference in arrival times between the two targets and elevating reaction by making the temporal discrimination more difficult. To determine whether both first-cued and second-cued RTs had been elevated late in the time period in which IOR is known to operate, a neutral baseline was included in Experiments 2 and 3, in which neither peripheral location was cued but, instead, a square drawn around the fixation point was brightened. In Experiment 2, beyond a $400 \mathrm{msec}$ cue lead time, RTs for both first-cued and second-cued double-target conditions were longer in this neutral condition. Hence, it was inferred that IOR was operating in first-cued but not in second-cued double-target trials.

An examination of the TOJ data from Experiments 2 and 3 revealed them to be similar to those from Experiment 1 . That is, there was no evidence of an inhibitory effect operating on the TOJs, but there was evidence of speeded signals overlapping partially with the timecourse in which IOR would be expected to operate. The Experiment 2 RT data were also similar to the double-target RT data from Experiment 1, with RT performance in the neutral condition being midway between slower second-cued and faster first-cued responding early in the timecourse and faster than both cued conditions late in the timecourse. Gibson and Egeth (1994) took this as evidence that IOR delayed arrival of the first-cued target and, hence, responding, whereas facilitation rather than IOR accelerated arrival of the second-cued target, thus slowing responding by making the discrimination more difficult.

To explain the hypothesized differential operation of IOR in the first-cued and second-cued conditions (as suggested by the RT measure) and to reconcile the repeat- 
edly observed failure to find an influence of IOR on perception in the double-target (TOJ) paradigm, Gibson and Egeth (1994) hypothesized that a new process, DOR, operates in such experiments. At the time of their reasoning, independent, preliminary evidence for DOR was provided by Pratt and Abrams (1993). It was postulated that DOR is a fast-acting process triggered by the appearance of stimuli at uncued locations and that DOR removes the inhibitory effects associated with IOR. Further, it was proposed that IOR has not been found to affect perceptual measures in the TOJ task because of the pre-emption of such effects by DOR.

Gibson and Egeth's (1994) reasoning included the following assumptions: first, that IOR inhibits perceptual representations, and second, that DOR, triggered by the appearance of subsequent stimuli, removes IOR. To escape criticisms that IOR is observed in the traditional paradigm, in which a central brightening mediates cue and target presentations (see, e.g., Posner \& Cohen, 1984), and that such a brightening apparently does not invoke DOR, the additional assumption was adopted that DOR only arises from stimuli presented at uncued task-relevant locations. Furthermore, some of their RT data (i.e., the absence of IOR in first-cued condition when the target asynchrony was $72 \mathrm{msec}$ or less) required Gibson and Egeth to make one further assumption: that inhibition encountered by a target can be removed by a stimulus that occurs up to $72 \mathrm{msec}$ after the target's appearance (i.e., T2 can remove inhibition at the T1 location for up to $72 \mathrm{msec}$ after $\mathrm{T} 1$ is presented; see p. 676). Since some amount of perceptual processing of the second target must take place before it can activate DOR, we find this proposal to be awkward for the assumption that DOR is designed to salvage: that IOR affects early perceptual processing.

\section{Response-Based Accounts of IOR Are Viable}

A simpler framework for coordinating the Gibson and Egeth (1994) data and previous TOJ work is provided by assuming (as did Klein \& Taylor, 1994; Maylor, 1985; Posner et al., 1985) that IOR affects response processes (response selection, response initiation). We propose that the RT delay associated with IOR arises primarily from the visual-motor system rather than through visual-perception processes (see Schmidt, 1996, for an elaboration of a variant of this account, first put forward by Tanaka \& Shimojo, 1993). Since this view holds that the primary locus of IOR is not within perceptual systems, IOR would not be expected to affect measures that only tap this level of processing (i.e., TOJs) but would be expected to influence response time (i.e., the localization RT data that Gibson and Egeth collected in their TOJ task).

Gibson and Egeth (1994) considered the possibility that a response-based account might explain their pattern of results. Their analysis showed that "some form of inhibition to respond to the cued location" (p. 677) could account for all but one of their RT findings (namely, that cued single-target RT was faster than first-cued double-target RT), and, on this basis, they rejected response inhibition as a plausible explanation for their set of data. However, precisely what form of response inhibition Gibson and Egeth had in mind is unclear from their paper.

One possible construal of response inhibition (see Klein \& Taylor, 1994, pp. 114-117, for a discussion of different categories of cognitive inhibition) is a process that prohibits a response from being made so long as the inhibition has not decayed or been removed. Under this interpretation, the magnitude of IOR would be maximal upon its commencement and would decrease on a millisecond for millisecond basis with increasing cue-target SOA. Because this construal is atypical within the IOR literature and is inconsistent with known properties of IOR (e.g., IOR is often less than $40 \mathrm{msec}$ and can last for several seconds), it will occur to few readers of the target article. Yet Gibson and Egeth (Gibson, personal communication, April 1997) did mean to propose, and reject, a response-based account of their data, in which a response could not be made until IOR had ceased. Because this view does not provide a viable account of the IOR data in the literature (and is inconsistent with several features of the target article's data, in addition to the comparison on which the rejection of one response-based account was based), we see it as a straw man, whose rejection should not taint the general class of response-based accounts of IOR.

A more common meaning of inhibition, and the one most readers will probably assume to have been intended by Gibson and Egeth (1994), is negative activation. Responses experiencing this type of inhibition can be executed, but only after sufficient positive activation accumulates to overcome the negative input. Inhibition of this sort is like a handicap that impedes or slows the inhibited mental operation. This construal of inhibition is not ruled out by the RT difference between cued single-target and first-cued double-target conditions, because any differences between these conditions are not necessarily eliminated by negative activation (as they would be under the prohibitive construal discussed above). Task difficulty (Gibson \& Egeth, 1994, p. 673) is one such difference: The task (i.e., Which target appeared first?) is more difficult when there are two targets than when there is only one, and decision time would be expected to reflect this. Additionally, the mere occurrence of a second stimulus on double-target trials may cause a RT delay, as compared with single-target trials, even without assuming a change in task difficulty (e.g., through response competition or some other process). For example, there is clear evidence of a such a delay in Müller and von Mühlenen (1996, Experiments $1,2,3$, and 4 ), in which a central flash at the 500-msec SOA significantly affected RTs to single targets presented 100-250 msec earlier. Hence, a negative activation interpretation of IOR is not refuted by the single finding that Gibson and Egeth used to reject their response inhibition account. ${ }^{2}$

Although a dissociation between RT and perception follows naturally from the assumption that IOR affects stages associated with responding, within the negative activation construal of IOR that we have advanced, it re- 
mains to be explained why IOR, as manifest by RT in the TOJ task, is sensitive to the intertarget interval (recall that, in their two target conditions, Gibson and Egeth [1994] obtained IOR in RT only when the intertarget interval was relatively long; see above). One explanation can be arrived at by applying Sternberg's (1969) additive factors logic: The finding that response competition (which is amplified when $\mathrm{T} 1$ and $\mathrm{T} 2$ are close together) interacts with IOR (IOR occurs at long intervals when conflict is minimal, but not at short intervals when it is maximal) can be interpreted to mean that IOR and response conflict affect at least one stage in common. Although, in principle, such a stage could be early or late in the processing sequence, we find it natural to assume that it is motoric. ${ }^{3}$

A second reason that IOR may have been found to vary with the intertarget interval in the target paper can be derived from Klein and Taylor's (1994, pp. 142-143) proposal that IOR is obtained when the subject's task involves responding toward a target location (detection or localization), whereas it is not obtained when the task involves making a decision on the basis of a nonspatial attribute (for the case in question, deciding which of two closely spaced stimuli appeared first). This proposal, which was based partly on the failure of several investigators to obtain IOR in a nonspatial discrimination task, is similar in its consequences to the assumption of DOR. However, according to DOR, the occurrence of subsequent stimuli removes (disinhibits) IOR, whereas, in the Klein and Taylor response/localization account, IOR may be present but is not observed when the decision concerning which response to make shifts from the spatial dimension (in this case, to the temporal dimension).

Finally, it should be noted that, if DOR, as described by Gibson and Egeth (1994), existed (the next section explains why this is unlikely), their pattern of findings could be accommodated, whether the inhibition that DOR removed was perceptual or motoric in nature. Hence, in the absence of any directly observable effects of IOR on perception (see Gibson \& Egeth, p. 672; Maylor, 1985; Posner et al., 1985; Schmidt, 1996), the target paper's favoring of an interpretation that IOR affects processes acting at a perceptual level is circular.

\section{Evidence Against Disinhibition of Return}

Whether it is assumed that IOR arises from degraded perceptual or motoric representations, Gibson and Egeth's (1994) framework provides equally viable accounts of their findings. So long as DOR is accepted as a viable process their framework also allows the view that IOR delays perception to escape the contradictory findings from studies of temporal perception (TOJs). In this section, we discuss empirical results that suggest that a process like DOR does not operate, and we present the results of a new experiment that directly failed to find support for the operation of such a process.

In recent studies, persistent $\mathrm{RT}$ inhibition in paradigms with multiple cues has been observed (Abrams \& Pratt, 1996; Danziger, Kingstone, \& Snyder, in press; Tip- per, Weaver, \& Watson, 1996; Wright \& Richards, 1996), which provides direct evidence against a DOR mechanism. Tipper et al. have shown that IOR can be observed at up to four locations cued in succession. Abrams and Pratt replicated these results and argued that Tipper et al. observed a spatially diffuse form of inhibition. Danziger et al. and Wright and Richards have also reported the ability to observe IOR at multiple previously cued locations. Each of these studies provides direct evidence against the operation of DOR, in which stimulation beyond the first cue is predicted to cancel IOR.

A second line of evidence against DOR and against Gibson and Egeth's (1994) foundational assumption that IOR reflects an impairment of perceptual processing comes from the illusory line-motion experiments of Schmidt (1996). If a line is presented to an observer all at once, with one end in the vicinity of a recently cued spatial location, the observer experiences illusory motion within the line away from the cued location, as if the line were being painted on over time. This illusory line-motion effect is believed to occur as the result of the signals arising from one end of an instantaneously presented line being transmitted more quickly than the signals arising elsewhere on the line, which causes a smooth percept of motion within the line, away from the initial source of signal transmission (Hikosaka et al., 1993). Hence, like judgments in the TOJ task, the illusion is believed to result from the prior entry of relative signals at a motion detection system (see Downing \& Treisman, 1997, for an attentional-biasing account of the illusion).

Schmidt (1996) investigated IOR with illusory linemotion by presenting a line probe, in order to assess the distribution of facilitative and inhibitory effects under conditions comparable to those in a conventional IOR paradigm. In one experiment, after cuing a potential target location, a line probe appeared between one of the two potential target locations and the fixation point, over a timecourse in which IOR should be detectable if it affects signal transmission within the visual-perceptual system. Such an inhibitory effect would result in a reversal of the illusion, causing motion towards the previously cued location, or, at a minimum, the nulling of the effect in line with the RT timecourse of inhibition. Cue lead times of $250,350,550,750$, and $1,150 \mathrm{msec}$ were examined in combination with target (a simple dot) asynchronies of 0 (no target dot presented, hence only the probe line appeared after the cue), 15, 30, or 45 msec. Under all of these stimulus conditions, which, for positive target asynchronies, were shown in a subsequent experiment to elicit IOR with a RT measure, only speeded perceptual processing (motion in the line away from the previously cued spatial location) was found.

In a similar second experiment, Schmidt (1996) presented the line probe between the two potential target locations. Again, there was no evidence of a perceptual impairment. It has been suggested that "Schmidt's illusory line motion experiments do not provide an adequate test of GE's claims ... [because] ... presenting a line that ex- 
tends from one (cued) location to another (uncued) location is similar to presenting one stimulus at the cued location and one stimulus at the uncued location (as in the TOJ task)" (Gibson, personal communication, September 1996).

To directly assess whether the probe line in Schmidt's (1996) Experiment $2^{4}$ was eliciting DOR (thus preventing IOR from reversing the direction of the illusion), we explored the impact of such a line on RT to targets in a typical IOR study (consult the Appendix for the full methods and results) in which a line accompanied the target on half of the trials. As in Schmidt's Experiment 2, a line was presented between the two potential target locations. The subject's task was to respond to the target as quickly as possible, whether or not an accompanying line was present. Gibson and Egeth's (1994) reasoning predicts that DOR should set in and eliminate IOR, as reflected by RT. The results showed only a significant $(p<.008)$ IOR effect of $17 \mathrm{msec}$ at a $700 \mathrm{msec}$ SOA. This direct test failed to support the proposal that DOR was elicited by Schmidt's line stimuli, thus preventing an arrival time delay due to IOR from occurring. This finding strengthens Schmidt's original conclusion that IOR does not, for the most part, result in impaired or delayed perceptual representations.

In summary, both of Gibson and Egeth's (1994) founding assumptions are problematic from an empirical view. Whereas Gibson and Egeth assumed that IOR impairs perceptual processing and hypothesized a new mechanism (DOR) to reconcile their observed dissociation between RT and TOJ data, illusory line-motion data demonstrates that these assumptions are unnecessary, particularly given that a response-based account of their data has not been ruled out.

\section{Conclusions}

Contrary to statements made by Gibson and Egeth (1994) in their abstract and conclusions, IOR has not been shown to affect TOJs. Had the qualifier the speed of been included, so that the abstract and conclusions read: "IOR affects the speed of TOJs," the opportunity for misunderstanding would have been greatly reduced. The data have demonstrated, in the context of a TOJ task, that IOR delays the time to respond under conditions in which temporal order is least ambiguous (single-target trials and the largest target asynchronies). When temporal order is difficult to resolve, this delay vanishes. There is no apparent delay in the speed of perceptual signal transmission or the inhibition of perceptual representations at a previously cued location, as revealed by the TOJ task, or in judgments of illusory line-motion (Schmidt, 1996). Furthermore, there is no evidence for the operation of DOR in several recent multiple cue experiments (Abrams \& Pratt, 1996; Danziger et al., in press; Tipper et al., 1996; Wright \& Richards, 1996), and our direct test (see Appendix) demonstrates a failure to observe DOR under conditions in which it was predicted to operate. Because Gibson and Egeth's data do not discriminate between a perceptual and a motor locus for IOR and because their framework is based on their assumption that IOR acts on perception and that DOR exists, it is at best circular for their "findings" to be cited as evidence for a perceptual level locus for IOR or for DOR.

There are four contributions of the target article that we would like to emphasize. Three are empirical. First, the new TOJ data constitute an impressive replication of Maylor's (1985) findings, despite the use of speeded responses. Although the target article alluded to the replication, it is easy to miss. One goal of our commentary was to highlight this contribution. The second contribution is the demonstration of a dissociation, within the same task, between the psychophysical (TOJ) data and some of the RT data. For those who believe that IOR is a responserelated phenomenon, the dissociation requires little explanation: Being motoric in nature, IOR is seen in the speed of motor responses (RT) to events but not in the perceptual arrival times of the very same events. A third contribution is Gibson and Egeth's (1994) discovery that IOR in RT varies with the interval between the two targets in a speeded TOJ task. Any theory of IOR must explain this pattern. Within the response-based framework we have advanced above, the failure of RT to show evidence of IOR when the two targets are closely spaced might be attributed to an interaction between response competition and response inhibition or to a shift in the representation used by the decision system to determine the response (i.e., from space to time, with only the former sensitive to IOR).

A fourth contribution of the target paper lies in Gibson and Egeth's (1994) proposed framework, which organized the seemingly contradictory RT and perceptual measures, in order to reconcile them with the assumption that IOR results primarily from impaired or delayed perceptual representations. Although creative and perhaps plausible when it was proposed, we have shown here why such a proposal is logically unnecessary and empirically unlikely.

Recently, a number of researchers have interpreted their RT findings as support for the idea that IOR acts at a perceptual level (see, e.g., Abrams \& Dobkin, 1994; Reuter-Lorenz, Jha, \& Rosenquist, 1996). ${ }^{5}$ This view must come to grips with the ubiquitous failure of perceptual measures, under normal circumstances, to show evidence of inhibited temporal processing in the perception of previously cued targets. If DOR can be shown to be an authentic process while also making sense of accumulating data that suggests otherwise, Gibson and Egeth (1994) have provided one way to resolve this conflict. The negative evidence we have reviewed here, however, suggests that a different rapprochement may be needed. One possibility is to assume that IOR may affect some perceptual operations or stages without delaying the arrival times on which TOJs are based. In addition, the view that IOR reflects primarily a motoric RT delay (Klein \& Taylor, 1994; Maylor, 1985; Posner et al., 1985; Schmidt, 1996) must come to grips with the possibility that there 
may be a perceptual component as well, although direct measures of such a component have not yet been found.

As with attention, IOR may have consequences at more than one locus in the processing stream. After expressing some skepticism about the ability of a narrow range of RT tasks to shed light on perceptual processes on which attention might act (because effects of attention can occur at any point in the sequence "from image encoding through response selection"), Shulman (1990) concludes: "It is increasingly clear that researchers in attention have only been partly successful in specifying the locus of attentional effects. One strategy for solving this problem is to embed attentional manipulations within perceptual paradigms that isolate particular computations" (p. 202). We strongly endorse Shulman's suggestion and believe that it has been and will continue to be a fruitful strategy when extended to IOR (as was done by Maylor, 1985, and Schmidt, 1996). Converging evidence from more than simple RT studies will be needed to fully explicate the nature of IOR.

\section{REFERENCES}

ABRAMS, R. A., \& DoBKIN, R. S. (1994). Inhibition of return: Effects of attentional cuing on eye movement latencies. Journal of Experimental Psychology: Human Perception \& Performance, 20, 467-477.

Abrams, R. A., \& PratT, J. (1996). Spatially-diffuse inhibition affects multiple locations: A reply to Tipper, Weaver, and Watson (1996). Journal of Experimental Psychology: Human Perception \& Performance, 22, 1294-1298

DANZiger, S., Kingstone, A., \& SNyder, J. J. (in press). Inhibition of return to successively stimulated locations in a sequential visual search paradigm. Journal of Experimental Psychology: Human Perception \& Performance.

Downing, P., \& Treisman, A. (1997). The line-motion illusion: Attention or impletion? Journal of Experimental Psychology: Human Perception \& Performance, 23, 768-779.

FORBES, K., \& KLEIN, R. M. (1996). The magnitude of the fixation offset effect with endogenously and exogenously controlled saccades. Journal of Cognitive Neuroscience, 8, 344-352.

GIBSON, B. S., \& EGETH, H. (1994). Inhibition and disinhibition of return: Evidence from temporal order judgments. Perception \& Psychophysics, 56, 669-680.

Hikosaka, O., Miyauchi, S., \& Shimojo, S. (1993). Voluntary and stimulus-induced attention detected as motion sensation. Perception, 22, 517-526.

KLEIN, R. M., \& TAYLOR, T. L. (1994). Categories of cognitive inhibition with reference to attention. In D. Dagenbach \& T. H. Carr (Eds.), Inhibitory mechanisms in attention, memory and language (pp. 113-150). San Diego: Academic Press.

KWAK, H.-W. (1992). Inhibitory and facilitatory components of orienting attention to locations and to features. Unpublished doctoral dissertation, Johns Hopkins University.

MAYLOR, E. A. (1985). Facilitatory and inhibitory components of orienting in visual space. In M. I. Posner \& O. S. M. Marin (Eds.), Attention and performance XI: Mechanisms of intention (pp. 189-204). Hillsdale, NJ: Erlbaum.

Müller, H. J., \& von Mühlenen, A. (1996). Attentional tracking and inhibition of return in dynamic displays. Perception \& Psychophysics, 58, 224-249.

Posner, M. I., \& COHEN, Y. (1984). Components of visual orienting. In H. Bouma \& D. Bowhuis (Eds.), Attention and performance $X$ (pp. 531-556). Hillsdale, NJ: Erlbaum.

Posner, M. I., Rafal, R. D., Choate, L. S., \& Vaughan, J. (1985). Inhibition of return: Neural basis and function. Cognitive Neuropsychology, 2, 211-228.

Pratt, J., \& ABrams, R. A. (1993, November). Inhibition of return: Ef- fects of multiply-cued spatial locations. Poster presented at the 34th Annual Meeting of the Psychonomic Society, Washington, D.C.

Rafal, R. D., Calabresi, P. A., Brennan, C. W., \& Sciotto, T. K. (1989). Saccade preparation inhibits reorienting to recently attended locations. Journal of Experimental Psychology: Human Perception \& Performance, 15, 673-685.

Reuter-Lorenz, P. A., JHA, A. P., \& Rosenquist, J. N. (1996). What is inhibited in inhibition of return? Journal of Experimental Psychology: Human Perception \& Performance, 22, 367-379.

SchмidT, W. C. (1996). Inhibition of return is not detected using illusory line motion. Perception \& Psychophysics, 58, 883-898.

Shulman, G. L. (1990). Relating attention to visual mechanisms. Perception \& Psychophysics, 47, 199-203.

Stelmach, L. B., \& Herdman, C. M. (1991). Directed attention and perception of temporal order. Journal of Experimental Psychology: Human Perception \& Performance, 17, 539-550.

STERNBERG, S. (1969). The discovery of processing stages: Extensions of Donders' method. In W. G. Koster (Ed.), Attention and performance II (pp. 276-315). Amsterdam: North-Holland.

SternberG, S., \& KNOLL, R. (1973). The perception of temporal order: Fundamental issues and a general model. In S. Kornblum (Ed.), Attention and performance IV (pp. 629-685). New York: Academic Press.

TANAKA, Y., \& SHIMOJO, S. (1993). Location priming and probability priming are isolatable in detection and discrimination. Investigative Ophthalmology \& Visual Science, 34, 1234.

TAYLOR, T. L., \& KLEIN, R. M. (in press). On the causes and effects of inhibition of return. Psychonomic Bulletin \& Review.

TIPPER, S. P., WEAVER, B., \& WATSON, F. L. (1996). Inhibition of return to successively cued spatial locations: Commentary on Pratt and Abrams (1995). Journal of Experimental Psychology: Human Perception \& Performance, 22, 1289-1293.

WRIGHT, R. D., \& RiChARDS, C. M. (1996). Inhibition of return at multiple locations in visual space. Canadian Journal of Experimental Psychology, 50, 324-327.

\section{NOTES}

1. These data were derived from Gibson and Egeths's (1994) published data by recovering the probability that the subject responded to the target appearing at the cued location and then applying a $Z$ transform to these probabilities. Error frequencies in second-cued conditions were used for positive target asynchronies (where the cued target lags the uncued target). Values for negative target asynchronies (where the cued target leads the uncued target) were derived from the first-cued condition data by assuming that, when subjects were not making errors in this condition, they perceived the cued target as occurring first.

2. According to Gibson (personal communication, June 1997), he and Egeth did consider a simple negative activation response-based explanation for their Experiment 3 results but dismissed it because IOR was not observed in the first-cued condition when the temporal gap between stimuli was short. Thus, although not explicitly stated in their article, it is likely that Gibson and Egeth would have rejected both prohibitive and negative activation versions of the response-based account. In the text below, and in note 3 , it is argued that negative activation response-based accounts of IOR can be fashioned that are consistent with Gibson and Egeth's (1994) findings.

3. The underadditive interaction that Gibson and Egeth (1994) observed could be explained by a mechanism in which the inhibition (of the negative activation variety) and facilitation of each response is accumulated by separate nodes, so long as the inhibitory node's output is asymptotic when highly activated. Alternatively, the underadditive interaction might be interpreted in terms of parallel processing, in which the motoric delay associated with IOR is absorbed into the extra time required to select and initiate a response when competition between responses is intense.

4. Note that DOR would not be expected to act in Schmidt's Experiment 1 , in which the probe stimulus was presented between the cued location and the task-irrelevant fixation. This experiment also failed to find any influence of IOR on perception. 
5. Abrams and Dobkin (1994) interpreted their RT data as suggesting that IOR was at least partly a perceptual impairment. Because their conclusion was emphasized in Gibson and Egeth (1994), we feel it is warranted to show that other interpretations are possible. In this study, one of two locations was cued, and subjects then were required to make a saccade to the cued or to the opposite location, in response to a peripheral luminance increment or to a centrally presented arrow. There was IOR in the arrow condition (strongly implicating a motor component), but the RT effect was three times larger in the peripheral target condition. Although this was interpreted to mean that two thirds of the inhibitory effect was in the detection of the target, this is not the only interpretation. Saccades in response to peripheral targets would naturally be programmed and executed under the control of the reflexive machinery of the superior colliculus. In contrast, programming of saccade metrics in response to an arrow at fixation would depend on machinery outside the colliculus (most likely, frontal mechanisms). Because the task involves looking away from the stimulus currently being processed, it also bears a resemblance to the antisaccade task, for which reduced colliculus involvement and, probably, inhibition of the colliculus are likely (Forbes \& Klein, 1996). If, as we believe (see Rafal, Calabresi, Brennan, Sciotto, 1989), the superior colliculus plays an important role in the generation of IOR and in its impact on subsequent processing, IOR will vary positively with the degree of collicular involvement--precisely what was found by Abrams and Dobkin.

\section{APPENDIX}

\section{Method}

Observers. Fifteen members of the university community were paid to participate in a 15 -min experimental session. All observers reported normal or corrected-to-normal vision.

Apparatus and Stimuli. The stimuli were presented on a Macintosh Color Plus display controlled by a Macintosh LC630 microcomputer running W.C.S.'s SplayPicts 1.1 experiment software package.

All of the stimuli presented were gray on a black background. A small fixation cross in the middle of the display (composed of two lines $0.2^{\circ}$ wide and $1.0^{\circ}$ long) and two empty boxes $\left(2.75^{\circ} \times 2.75^{\circ}\right)$ drawn $0.5^{\circ}$ above fixation, $5.5^{\circ}$ on either side of the display's midline, marked potential target locations and were present throughout the trial. Cuing was accomplished by increasing the width of a box's border (from $0.1^{\circ}$ to $0.3^{\circ}$ ). Targets consisted of $1^{\circ} \times 1^{\circ}$ filled squares centered in either the left or right box. A centered line, $0.2^{\circ}$ high and $10.5^{\circ}$ long, all but joined the potential marker locations.
Procedure. Each observer sat in a dimly lit room at a distance of $57 \mathrm{~cm}$ from the computer monitor, with his or her head being steadied by a chinrest. On every trial, the fixation cross was presented for $1,000 \mathrm{msec}$, followed by the cue for $100 \mathrm{msec}$. Targets or targets and a line were presented with a $750 \mathrm{msec}$ SOA. On standard IOR trials, a target appeared in one of the two boxes. On target plus line trials, a line between the boxes accompanied the target. On catch trials, no target appeared and either a line appeared (line catch) or nothing happened after cuing. The observer's task was to respond as quickly as possible upon detecting a target by pressing the computer keyboard's spacebar. If no target was presented, the observer was instructed to withhold a response, and the session proceeded after $2,000 \mathrm{msec}$. RTs accurate to the nearest millisecond were recorded. Breaks were enforced every 50 trials, and the subject's response triggered the beginning of the next trial after a $1,000 \mathrm{msec}$ intertrial interval.

Design. The design was 2 (cue validity, valid or invalid) $\times 2$ (line status, present or absent) $\times 2$ (cue side, left or right). There were 160 experimental trials ( 20 observations per cell), 40 catch trials ( 20 presented with a line and 20 without), and 20 practice trials (randomly selected from the entire corpus), for a total of 220 trials per observer.

\section{Results and Discussion}

For each observer, the mean RTs for trials in each condition that fell in the range of $100 \mathrm{msec}$ to 2.5 standard deviations above the mean RT without exclusions were analyzed. Responses with RTs below $100 \mathrm{msec}$ were considered anticipations, and those with RTs above $1,500 \mathrm{msec}$, outliers. A 2 (cue validity) $\times 2$ (line status) $\times 2$ (cue side) repeated-measures ANOVA was carried out on the observers' mean RTs.

The only significant effect was of cue validity $[F(1,13)=$ 9.82, $M S_{\mathrm{e}}=8,169.57, p<.008$ ], indicating an overall IOR effect of $17 \mathrm{msec}$ (a valid RT of $396.3 \mathrm{msec}$ vs. an invalid RT of $379.2 \mathrm{msec}$ ). Although there was not a significant cue validity $\times$ line status interaction $\left[F(1,13)=0.92, M S_{\mathrm{e}}=271.83, p>.35\right]$, IOR was $6 \mathrm{msec}$ stronger when a line was present $(20 \mathrm{msec})$ than when absent $(14 \mathrm{msec})$. All remaining effects were nonsignificant.

(Manuscript received September 19, 1995; revision accepted for publication July 18, 1997.) 